\title{
Théologiques
}

Revue interdisciplinaire d'études religieuses

\section{La fragilité dans la culture et les attentes spirituelles de l'individu contemporain}

\section{Marc Leboucher}

Volume 28, numéro 1, 2020

Pour une culture palliative ? Fragilités et vulnérabilité

URI : https://id.erudit.org/iderudit/1074674ar

DOI : https://doi.org/10.7202/1074674ar

Aller au sommaire du numéro

Éditeur(s)

Institut d'études religieuses de l’Université de Montréal

ISSN

1188-7109 (imprimé)

1492-1413 (numérique)

Découvrir la revue

\section{Citer cet article}

Leboucher, M. (2020). La fragilité dans la culture et les attentes spirituelles de l'individu contemporain. Théologiques, 28(1), 17-41.

https://doi.org/10.7202/1074674ar

\section{Résumé de l'article}

Si le concept de fragilité prend tout naturellement sa place dans une réflexion d'ensemble sur le soin, il reste qu'il trouve des illustrations dans d'autres secteurs de la culture contemporaine. Écrivains, peintres ou musiciens traduisent à leur manière des attentes spirituelles d'aujourd'hui. Après avoir précisé la notion d'attente spirituelle en lien avec l'inquiétude actuelle, il s'agit ici d'interroger l'oeuvre de trois artistes pour mieux les relier à des aspirations plus larges. Si la romancière Sylvie Germain aide à mieux percevoir de telles attentes en termes de reconnaissance, d'écoute et d'éternité de la part des individus d'aujourd'hui, les toiles et la vie de Nicolas de Staël aident de leur côté à discerner celles-ci en termes de dépassement de soi, de corporéité et de gratuité. Le destin du pianiste Glenn Gould aide enfin à mieux percevoir ces demandes en termes de ritualité, de relation à la nature et de nomination du divin qui s'expriment désormais. 


\title{
La fragilité dans la culture et les attentes spirituelles de l'individu contemporain
}

\author{
Marc LEBOUCHER* \\ Éditeur et écrivain \\ Paris
}

Avant de devenir un mot très largement employé, c'est d'abord dans le domaine de la santé, en particulier celui de la néonatalogie, du handicap et de la gériatrie, du début et de la fin de vie, que le mot de fragilité a trouvé tout naturellement sa place. Dans son ouvrage Fragilité, le philosophe JeanLouis Chrétien remarque que parmi les images les plus souvent utilisées à son sujet au cours des siècles, le roseau, le verre, la bulle de savon ou les ruines, celles du nourrisson ou du vieillard se retrouvent de manière fréquente (Chrétien 2017). On ne s'étonne donc pas de voir le mot faire florès actuellement dans l'univers du soin, à travers des expressions comme celle de syndrome de glissement, de détresse ou de faiblesse. Il renvoie du même coup à la question de la dignité humaine et aux actuelles interrogations éthiques.

D'emblée, un consensus transdisciplinaire se dégage pour distinguer la fragilité de la vulnérabilité, la première relevant de la structure interne à une personne, à un corps voire à un système, la seconde tenant davantage à une atteinte venant de l'extérieur. En témoignent ainsi les définitions que nous donnent Gaëlle Fiasse $(2015,10)$ - est vulnérable ce qui peut être

* Né en 1959, Marc Leboucher est éditeur aux éditions Salvator à Paris et écrivain. Après des études de droit et de sociologie politique, il a travaillé dans un service de la Conférence des évêques de France, comme journaliste au mensuel Panorama, puis comme directeur littéraire et président des éditions Desclée de Brouwer. Particulièrement intéressé par les liens du christianisme et de la culture, il a publié des livres entretiens avec Joseph Moingt, Bernard Sesboüé et René Rémond, et en 2017, un ouvrage consacré à la fragilité sous le titre Le souffle et le roseau. Variations sur la fragilité, Salvator, Paris, 2017. 
facilement atteint, blessé — ou Corine Pelluchon pour qui « la vulnérabilité fait référence à notre susceptibilité au plaisir, à la douleur et au temps, à l'incomplétude du psychisme et souligne à la fois notre besoin de l'autre et notre ouverture à l'autre»(Pelluchon 2011, 282-283). Pour Philippe Kourilsky $(2019,19)$, la robustesse d'un système, soit en science soit même à travers le fonctionnement de nos démocraties, se mesure à la capacité à résister à des attaques externes ou internes, celui-ci se révélant capable de continuer à fonctionner en dépit de celles-ci. Pour s'en tenir à une définition plus commune, plus empirique, je citerai la réaction spontanée d'une participante à l'issue d'une conférence sur ce thème :

À mon âge, à près de soixante-dix ans, je suis bien sûr vulnérable, je peux à tout instant être victime d'un accident ou d'une crise cardiaque, tomber malade... Pour autant, je ne me sens pas fragile. J'ai l'impression de maitriser ma vie. Il en irait tout autrement si j'étais dépressive, par exemple. (Entretien avec l'auteur)

Mais désormais, à côté d'une production éditoriale abondante en sciences humaines et en spiritualité (Arènes 1999; Benasayag 2004; Carrière 2007; Jollien 2011; Ringlet 2007 ; Salenson 2016), la thématique de la fragilité déborde largement l'univers de la santé. Tout autant que médecins, philosophes ou sociologues, les écrivains, peintres, sculpteurs ou musiciens nous parlent de ce trait de l'homme contemporain (Leboucher 2017, 29). Si la notion de faiblesse renvoie dès l'Antiquité à la notion de finitude, à la condition mortelle, si le christianisme enrichit celle-ci d'une dimension morale à travers le thème du péché, du caractère faillible de l'homme comme le remarque Jean-Louis Chrétien déjà cité, elle revêt une autre nature dans la culture d'aujourd'hui. Quand Alberto Giacometti représente L'homme qui marche à travers une silhouette émaciée en mouvement (Maubert 2016), quand Francis Scott Fitzgerald évoque La fêlure dans un saisissant récit, quand Maurice Ravel écrit un Concerto pour la main gauche à l'intention du pianiste Paul Wittgenstein devenu manchot durant la Grande Guerre, n'illustrent-ils pas cette précarité de l'individu, tout autant ébranlé de l'intérieur que par les secousses de l'histoire, et qui se voit en même temps invité à un dépassement de soi ? On pourrait bien sûr s'arrêter là, et cela serait déjà beaucoup, les expressions artistiques se suffisant par elles-mêmes. Mais il faut se demander si, au-delà de la seule expérience de l'artiste, ces œuvres de culture ne révèlent pas aussi des aspirations, des demandes d'ordre spirituel plus largement partagées. 
Si nous faisons ici le choix d'une démarche d'anthropologie culturelle dont les frontières épistémologiques peuvent sembler parfois un peu floues, c'est bien parce que ces artistes et leurs œuvres témoignent non seulement d'une expérience personnelle, subjective mais qu'ils savent plus largement trouver écho auprès d'un public à un moment donné, rejoignant ainsi des attentes qui peuvent nourrir des réflexions esthétiques et philosophiques. Ces dernières doivent permettre aussi des ouvertures bienvenues en matière de théologie spirituelle.

Dès 1910, Wassily Kandinsky invite à cet élargissement du regard. Pour celui-ci, «la nécessité intérieure» de l'artiste «nait de trois raisons mystiques». Ce qui est propre à la personnalité du créateur, ce qui est propre à l'époque où il vit, à son langage ou à sa nation, ce qui est propre à l'art en général et transcende les époques (Kandinsky 1989, 132-133). Plus d'un siècle après, il n'est donc pas absurde d'interroger à sa suite quelques artistes d'aujourd'hui, pour mieux comprendre le langage et les attentes spirituelles de notre temps. Disant cela, il ne s'agit pas de les récupérer de manière réductrice dans le champ du religieux, mais de percevoir ce qu'ils révèlent plus profondément d'une quête intérieure.

Avant de se livrer à cet exercice, un constat et quelques précisions s'imposent.

\section{Un cœur sans repos}

En ouverture de ses Confessions, saint Augustin pointait d'emblée le « cœur inquiet» de l'homme éloigné de Dieu et qui ne peut trouver son repos qu'en lui (Augustin 1964,15). Nous ne sommes plus à l'époque de l'évêque d'Hippone, et pourtant il est tentant de reprendre à notre compte l'expression d'inquiétude qui en jaillit. Aujourd'hui, dans notre monde sécularisé où la référence à Dieu ne va plus de soi, où nous n'employons plus les mots de la foi, combien croisons-nous pourtant de "cœurs sans repos »! La quête spirituelle, l'aspiration à un "être plus » n'est-elle pas présente un peu partout? Des cœurs « chasseurs solitaires » ou «brisés », blessés ou déprimés, soumis aux «intermittences» ou aux émotions de tous ordres, n'est-ce pas le lot commun de notre humanité en quête de sa vérité propre?

Si beaucoup voient dans la pensée d'Augustin une étape essentielle dans la naissance de la constitution du Moi moderne, à l'instar de Charles 
Taylor (1998, 173), c'est sans doute aussi justement parce que nous continuons d'éprouver, même de manière confuse, cette inquiétude du « cour altéré », cette errance du Moi dont parle l'introspection augustinienne. Bien que privée de toute connotation religieuse, de l'invocation du « repos en Dieu », cette formulation intime demeure pour nous évocatrice. Même s'il semble souvent sûr de lui, indifférent aux questions de sens ou exclusivement tendu vers sa quête d'épanouissement solitaire, l'individu de notre siècle ne peut en effet cacher sa propre fissure intérieure dans son affrontement au mal, à la maladie ou à la mort.

Comment mieux préciser alors ce que nous entendons par l'expression «d'attentes spirituelles »?

Il s'agit d'abord de mieux définir le mot «attente ». La frontière se révèle ténue entre la notion d'attente et celles de demande, de besoin, de désir. On sait combien la satisfaction des besoins primaires ou secondaires préoccupe à juste titre les soignants ou les éducateurs dans leur démarche auprès des personnes en situation de fragilité. Comment distinguer alors entre la simple demande, le besoin exprimé et susceptible d'être satisfait, et l'attente plus profonde qu'il exprime, voire le désir intérieur? Ainsi du mot « soif ", qui peut tout autant désigner la demande d'être abreuvé que l'attente mystique de Dieu. Pour le philosophe Frédéric Laupies, «Les vivants ont des besoins : ils portent en eux, qu'ils en aient conscience ou non, des manques dont le comblement est vital. Mais l'être qui désire est le seul être capable de viser le manque en tant que tel " (Laupies 2019, 14). Dans cette perspective, il nous semble que l'attente se situe au-delà du simple besoin, dans une dynamique de désir authentique. Elle suppose patience et espérance, capacité à se projeter plus loin que l'instant présent. Elle invite à cette capacité d'attention chère à Simone Weil. Dans ce cadre, l'attention est tout aussi mobilisée chez celui qui attend, tendu vers l'avenir auquel il aspire, que chez l'autre à ses côtés, attentif à ce désir profond, dans ce mouvement de vulnérabilité circulaire bien mis en valeur par Jean-Marc Barreau (2017). Et puis, le désir renvoie au rapport ontologique à l'autre comme sujet de mon désir. On mesure ainsi toute la richesse d'un tel mot.

Attente donc, mais d'ordre spirituel. Comment appréhender cette dimension du «spirituel» dans un monde multiculturel où se croisent dans une confusion croissante valeurs et idéaux les plus variés, sagesses et religions en tous genres, indifférence totale ou dogmatisme le plus intransigeant? Dans un contexte où bien souvent les mots ou les lieux même 
manquent pour exprimer une telle demande? Sans être exhaustive, la définition proposée par le sociologue Jacques Grand'Maison nous paraît la plus adéquate pour nourrir notre réflexion. «Le spirituel [écrivait-il] c'est ce qui vient du plus profond de soi et qui, en même temps, nous dépasse. Risquerai-je la formule “d'en deçà de soi et d'au-delà de soi” ? Le spirituel, c'est donc ce qui donne profondeur à notre vie, à nos expériences humaines, à nos convictions et croyances, à nos amours, à notre foi en nous-mêmes, aux autres, et à l'avenir, et à Dieu pour ceux qui y croient » (Grand'Maison 2013, 13). Certains pourront trouver cette définition un peu trop extensive, mais elle a l'avantage de permettre à nombre d'entre nous de s'y retrouver, quelle que soit son appartenance ou ses convictions personnelles.

Enfin, sans reprendre ici les multiples analyses consacrées à la sécularisation de nos sociétés, rappelons que ces aspirations spirituelles ne se déploient plus dans un cadre religieux traditionnel. Dans un article prémonitoire de la revue Christus qui fit du bruit à l'époque, François Roustang signalait l'émergence dans le catholicisme des années soixante d'un « troisième homme ", témoin d'une nouvelle sensibilité religieuse (Roustang 2019). Selon lui, cet individu issu de la modernité ne se déterminait plus en fonction d'une hétéronomie, de l'institution ecclésiale, en particulier mais par lui-même, dans une démarche désormais autonome. Du coup, les débats internes aux religions n'avaient plus d'intérêt pour lui, il quittait discrètement les Églises instituées. Il allait désormais se déterminer seul dans le domaine moral ou éthique, comme pour sa vie sexuelle par exemple. Nous sommes bien nous aussi dans ce temps du «troisième homme ", quelques décennies plus tard. Certes, certains continuent de fréquenter les Églises, mais il reste que chacun conduit d'abord par lui-même sa propre existence, voire « bricole » sa propre croyance. Si autrefois les attentes spirituelles passaient largement par le canal des seules religions instituées, il n'en va plus de même aujourd'hui. Il faut donc un spectre beaucoup plus largement ouvert pour les évaluer, bien au-delà des approches verticales, dans une appréhension culturelle en réseaux.

C'est à partir de l'expérience de trois artistes, Sylvie Germain, Nicolas de Staël et Glenn Gould qu'il nous faut poursuivre cette évaluation et faire émerger quelques attentes saillantes. Commençons par celle de la romancière française Sylvie Germain, au regard aiguisé sur l'individu de ce temps. 


\section{Sous le signe des parias}

Comment parler d'attente spirituelle sans évoquer d'abord la soif infinie de reconnaissance qui habite les individus d'aujourd'hui ? Certes, et l'on aura l'occasion d'y revenir, cette soif n'est pas dénuée d'ambiguité, qui peut souvent s'expliquer par une forte revendication narcissique ou identitaire. Mais si tant d'individus expriment une telle demande, n'est-ce pas que trop souvent ils ont le sentiment de ne plus exister, de disparaitre de l'attention et de la sollicitude de leurs semblables?

Parmi les romanciers contemporains, Sylvie Germain figure parmi ceux qui nous questionnent le plus fortement sur cette attente de reconnaissance à travers une œuvre foisonnante dont témoignent, entre autres, ces grands romans que sont Le livre des nuits (Germain 1985) et Jours de colère (Germain 1989). Ne se contentant pas de réfléchir sur la thématique de la fragilité ou sur le statut des personnages dans la création littéraire, elle n'hésite pas à mettre l'accent sur les frontières de notre monde - qu'elles soient mentales, sociales, imaginaires ou anthropologiques — pour tirer de l'oubli ou de l'indifférence tous ces êtres que nous laissons de côté. Tout est une question de regard semble nous dire en substance l'écrivain qui a consacré l'un de ses travaux universitaires à ce thème cher à la pensée d'Emmanuel Lévinas, tout se joue dans la manière dont nous continuons ou non de découvrir ces visages qui nous environnent. On sait combien pour Lévinas le visage, au-delà même de sa signification première, exprime la vulnérabilité de l'autre et invite à la responsabilité à son égard. Dans deux courts romans qui touchent au fantastique, L'inaperçu (2008) et Hors champ (2009), Sylvie Germain met ainsi en scène des personnages qui s'effacent, au sens propre, aux yeux de leurs contemporains, simplement parce que les autres les ignorent ou les rejettent, leurs proches en premier lieu. Familière de longue date des pays de l'Est, cette auteure a su observer également d'une manière aiguë ces marginaux qui peuplent nos sociétés, tous ces laissés pour compte sur lesquels personne ne s'arrête. Dans son récit Cracovie à vol d'oiseaux, relevons simplement cette poignante évocation d'un couple de SDF, saisis sur le banc d'un parc de Cracovie, dans la Pologne postcommuniste :

Ce jour-là, une fin d'après-midi d'été, quelques passants font la pause dans le jardin du Planty. Un couple dort, enlacé sur un banc. Ce ne sont pas deux jeunes gens, en goguette amoureuse, tendrement serrés l'un contre l'autre. C'est un couple sans âge, ou plutôt de cet âge confus, tout chamboulé ou 
chiffonné, celui que façonne la misère, la vodka frelatée, les mauvais coups du sort et dans la gueule.

Ils sont échoués sur la banquette de bois tels deux phoques; deux phoques dotés de membres assez désarticulés. Une jambe pend dans le vide, une autre traîne par terre, une troisième est repliée, une autre encore est appuyée contre le dossier. Quant aux bras, ils sont jetés dans tous sens. La tête de la femme est plaquée contre le torse de l'homme et lui, à moitié enfoui sous la tignasse d'un gris crasseux de sa compagne, a un œil fermé et le second entrouvert. Un filet de regard glauque, néanmoins un brin hargneux, filtre entre ses paupières gonflées. Il grogne dans son demi-sommeil. Il a raison, il est indécent de reluquer les gens à leur insu, dans leur intimité. Ce couple est chez lui ici, étendu dans son lit de fortune. Il n'en a sûrement pas d'autre, de lit, il improvise chaque jour sa maison au gré des rues, des cours, des squares, selon le temps et selon l'humeur dictée par la faim, la fatigue, l'ivresse. Car si on a cessé de transformer les meubles en podiums où beugler des slogans et de convertir des appartements en médailles pour ouvriers méritants, il reste des naufragés. Chaque système fabrique ses parias. (Germain 2000, 16-17)

Qui dira le désir de reconnaissance de cet homme et de cette femme? Comment se laisser toucher par leur regard, même glauque? Il fallait bien l'œil d'une artiste pour les tirer de cet oubli...

\section{Reconnaissance, écoute, éternité}

« Je ne suis pas reconnu ! Je n'existe pas à ses yeux! » Combien de fois n'entendons-nous pas cette revendication exprimée autour de nous, tant sur le plan personnel qu'au niveau plus collectif? À travers cette demande qui semble comme le préalable à toute discussion, toute poursuite d'une relation plus approfondie, l'individu manifeste qu'il entend être reconnu pour ce qu'il est dans sa singularité, sa condition propre. Au fond, j'existe parce que quelqu'un d'autre le veut bien et l'accepte. Parce qu'il va pouvoir me regarder, faire plus attention à moi, et surtout prendre en compte mes mérites ou, davantage, ma propre souffrance.

Cette soif d'être reconnu dans son être, voire son mal-être, on la voit s'exprimer de la manière la plus tragique à travers le phénomène des suicides. "Sous l'uniforme, la fragilité » titrait ainsi un quotidien français en 
évoquant les suicides de policiers qui se sont multipliés en France ces dernières années. Certes, le phénomène de mort volontaire interrogeait déjà le sociologue Émile Durkheim au XIXe siècle (Durkheim 1897). Mais la nouveauté est qu'il se produit désormais au sein une catégorie professionnelle censée incarnée la loi et l'autorité (Leboucher 2019, 53). Paradoxe déstabilisant de voir ainsi vaciller la force et la sécurité sur lesquelles on pensait pouvoir compter. Comme on l'a constaté dans d'autres secteurs d'activité, telles les entreprises de télécommunication en proie à de profonds bouleversements internes, les suicides se passent la plupart du temps sur le lieu de travail, témoignant d'un malaise, d'une souffrance au cour de l'exercice d'une profession. Si autrefois on mettait en avant, de manière presque péjorative ou moralisante, le «déséquilibre » d'une personne, son " instabilité » ou sa simple « dépression », aujourd'hui le langage se fait plus compatissant, qui entend comprendre les raisons d'une telle fragilité intérieure. On entend ainsi les syndicats de personnel ou les représentants de l'administration concernée se situer aussi dans ce registre tout en pointant également des conditions de travail devenues plus difficiles. De la part des agents qui mettent fin à leurs jours, il s'agit bien de manifester qu'une demande de reconnaissance n'a pas été honorée, que l'on n'a pas su entendre une souffrance et, surtout, la percevoir comme telle. L'enjeu est si fort que l'on est prêt à mettre sa vie en balance pour cela.

Au-delà des situations individuelles, cette demande de reconnaissance s'exprime aussi à travers les attentes d'une catégorie, d'une communauté ou d'un groupe. Nous expérimentons sur le plan collectif une véritable "lutte pour la reconnaissance ", selon la formule d'Axel Honneth (2000), non pour acquérir du pouvoir ou du «capital réel, économique » mais du "capital symbolique ». Il en est ainsi de la situation des minorités, qu'elles soient ethniques ou sexuelles, qui ont l'impression de ne pas être traitées comme elles devraient être, et se sentent exclues ou considérées comme mineures. Bien souvent, cette attente se double d'une dimension identitaire. Pensons également à la situation des victimes, celles par exemple de la pédophilie dans l'Église. Elles témoignent elles aussi d'un fort désir de reconnaissance, là encore de leur souffrance, mais également de réparation. Là aussi, le traumatisme conduit à une demande spécifique. Mais comment réparer ce qui peut apparaître infini, trop profond, définitif? 
Cette puissante attente en termes de reconnaissance suppose que soit honorée du coup une seconde attente, celle d'être écouté. Car le «cœur sans repos ", en recherche de paix ou de sens peut-être, a besoin de parler, de s'exprimer, de prendre la parole. Là encore revient le mot de «confession » déjà évoqué à propos d'Augustin, mais qui n'est pas d'abord l'aveu d'une faute ou d'une culpabilité, voire l'expression d'une conviction religieuse, mais bien le souci préalable de se dire, en des mots qui poussent à aller plus loin.

On rejoint ici ce sens de l'écoute qu'un Maurice Bellet avait particulièrement valorisé dans les divers aspects de sa recherche, écoute première ou simplement amicale, écoute du thérapeute ou du psychanalyste, voire de l'accompagnateur spirituel. Ce lieu à la fois ouvert et confidentiel va permettre à ceux qui traversent « l'en bas » de se confier. Avant toute chose, il s'agit d'accueillir l'humain dans toute sa densité, sans jugement préalable. «Vous commencerez par le respect » aimait à dire Maurice Bellet. "L'écoute pure, comme telle, va droit à l'être. En elle se fait ce don : le don d'être là, d'exister, de pouvoir être là par la parole, qui est tout l'être se donnant à entendre. L'écoute est, pour qui la goûte, venue à l'être » (Bellet 1989, 23). Elle appelle donc celui qui la pratique à une profonde éthique et attention. Il s'agit de se détourner de toute manipulation. Écouter ne signifie pas plaquer sur l'autre ses propres fantasmes, encore moins ses désirs de pouvoir, mais bien d'équilibrer sens de la bienveillance et juste distance. Pour l'accompagnateur spirituel, il s'agira de l'écoute en l'autre du souffle de l'Esprit.

Avec des mots qui ont gardé toute leur force quelques décennies plus tard, Maurice Bellet décrit cette attente de tous ceux qui sont en manque d'être écoutés, "parias » à leur manière, même si ce ne sont plus simplement ces SDF dont nous parle Sylvie Germain. "Misère du langage des pauvres de notre temps, c'est-à-dire des déracinés quand ce qui faisait leur dignité a disparu. Misère de ceux qui viennent au moment critique où tout ce qui faisait leur demeure s'est effondré; en sorte que, si riches soient-ils ailleurs de savoir ou savoir-faire, ils sont, en ce point décisif de leur passage, sans rien, sans même de quoi dire la détresse et l'absence » (Bellet 1989, 26).

Mais à l'instar de la demande de reconnaissance, ce désir d'écoute paraît bien souvent difficile à combler. Il est paradoxal de constater que 
même si nombre de traditions spirituelles ont à cœur de valoriser cette dimension, même si beaucoup de cellules psychologiques fleurissent à l'occasion d'une souffrance récurrente ou d'un drame collectif, nos contemporains ont toujours le sentiment de ne pas être assez entendus. Faut-il y voir le débordement d'un trop-plein d'émotions, d'une affectivité qui ne trouve pas l'occasion de s'exprimer suffisamment? Faut-il incriminer un Moi par trop narcissique? Ou peut-être l'absence le plus souvent de lieux d'expression authentique où l'on n'en reste pas justement au seul ressenti, où l'on peut prendre un peu de distance face à celui-ci et s'ouvrir à un questionnement spirituel qui va déplacer la demande initiale ? En ce sens, les accompagnateurs des personnes en fin de vie sont bien placés pour dire combien cette attente d'écoute a du sens. Et ce jusqu'au bout.

Car ces attentes de reconnaissance et d'écoute vont plus loin que de simples revendications du Moi. Elles traduisent plus profondément encore un désir de vie en plénitude, un souci de refuser une existence au rabais et insignifiante. Car pour tous ces souffrants, il s'agit bien de durer, d'une certaine manière, de se survivre mieux et autrement. De là à parler de souhait d'immortalité ou de désir d'éternité, il n'y a qu'un pas que certains sont prêts à franchir sans difficulté. Cette attente se traduit paradoxalement de deux manières, soit en refusant de disparaître soit justement en préférant le néant.

En mettant en avant les possibilités infinies que nous offrent sciences et techniques, la médecine, la chirurgie comme l'informatique ou l'intelligence artificielle, les tenants du transhumanisme illustrent à leur manière la première attitude, qui entend répondre à cette demande d'immortalité. Certes, il ne s'agit pas de l'éternité des philosophes ni du Paradis des religions, mais d'un vrai refus de disparaître dans l'immédiat en se prolongeant le plus longtemps possible. Cette utopie qui entend dépasser la fragilité et la précarité du corps souligne combien notre désir de vivre est insatiable. Désir d'être sans limites, fût-ce celle du temps et de la finitude.

A contrario, le débat sur la question des soins palliatifs et de l'euthanasie en témoigne, nombre de nos contemporains réclament un droit à « mourir dans la dignité » qui devient peu à peu une aide au suicide. Pour cette seconde attitude qui se pare d'une morale néo-stoïcienne, il faudrait mourir sans souffrir, disparaître avec sagesse, prendre sur soi d'en finir, car se dégrader, devenir vulnérable serait honteux, non seulement pour soi mais aussi pour l'entourage. Mieux vaut plonger dans le néant que de vivre 
fragilisé. On peut élargir ce constat à la pratique de la crémation, vivement encouragée pour des raisons sociales ou commerciales : s'il peut exister des motivations religieuses ou philosophiques parfois (se fondre dans un grand Tout, dans une démarche panthéiste ou d'inspiration hindouiste, par exemple), il y a surtout dans cette attitude la volonté de s'effacer, de disparaître là aussi plus vite, d'accélérer le processus de décomposition du corps. Au nom de la volonté de ne pas gêner les proches, on en vient à leur infliger un traumatisme supplémentaire, comme s'il y avait une seconde mort par ce mode de destruction du corps, volontaire cette fois.

Passons à présent à un second artiste, Nicolas de Staël, et à ce qu'il révèle des attentes spirituelles contemporaines.

\section{Un peintre entre foi et le vertige}

Parce qu'il a peint nombre de paysages de Méditerranée, on imaginerait volontiers la tombe du peintre Nicolas de Staël (1913-1955) dans quelque cimetière marin, entre océan et cyprès. Plus modestement, il faut la trouver dans l'un des lieux les moins esthétiques de Paris, au cimetière de Montrouge en périphérie de la capitale, bien loin du romantique Père-Lachaise ou des sépultures provençales. Mais qui peut oublier que l'artiste a représenté également à plusieurs reprises les footballeurs du Parc des Princes, symbole du Paris populaire, dans une débauche de force et de couleurs? S'il est impossible en quelques lignes de ramasser une existence tourmentée qui s'achève brutalement à 41 ans par un suicide, d'évoquer une œuvre en perpétuelle tension entre l'art abstrait et l'art figuratif, comment ne pas voir dans cette grande figure un témoin de notre propre fragilité?

Le destin de Nicolas de Staël croise très tôt les fractures du XXe siècle. Né à Saint-Pétersbourg d'une grande famille aristocratique russe, il doit fuir tout jeune avec les siens lors de la Révolution de 1917, avant de se réfugier en Pologne, puis en Belgique. Orphelin, élevé dans un collège jésuite de Bruxelles, il se tourne vite vers la peinture. Mais la reconnaissance de son travail (tout à la fois influencé par ses contemporains tel Vlaminck mais aussi par les maitres flamands Vermeer ou Rembrandt) viendra bien tard, au terme d'une courte carrière, lorsque collectionneurs et galeries s'intéresseront à lui pour lui offrir un succès international. Point de sérénité chez lui, de progression tranquille, de réussite assurée. À côté d'une vie sentimentale pleine de déchirures, Staël ne cesse de produire et en même temps 
de détruire ce qui ne le satisfait pas. Il y a chez lui une quête perpétuelle d'une forme d'absolu qui le hante.

Plus que d'autres critiques sans doute, Stéphane Lambert (2015) a bien senti le drame intérieur qui l'habite dans un court essai suggestif dont nous reprenons ici quelques passages. Sans voir dans son œuvre une démarche à dimension explicitement confessionnelle ou religieuse, il perçoit dans celle-ci une manière de se libérer de l'inquiétude d'exister. Un cour sans repos, là encore ? Lorsque Nicolas de Staël peint La table de l'artiste et La cathédrale, bloc d'où semblent se détacher d'étranges silhouettes à forme de statues, c'est pour lui l'occasion de rendre « hommage à l'homme en sa petitesse et en sa tragique condition capable par la force de l'esprit de se faire bâtisseur de cathédrales » (Lambert 2015, 140). Mais plus encore que cette conscience de notre humaine précarité, Yves Lambert pointe l'extrême tension qui anime l'artiste entre « foi et vertige » : «[...] c'est dans le vertige, tentait-il encore de penser, comme s'il essayait de reconstruire mentalement ce qui l'avait animé en tant que peintre, c'est dans le vertige que l'artiste tire la matière de son ouvre et c'est par la foi qu'il trouve la force de l'engendrer » (Lambert 2015, 34). Nous sommes là loin d'une approche de l'art, de la création issue d'une forme d'harmonie : chez Staël, « [...] ce qui faisait la grandeur de l'art, en ceci j'entendais l'intensité de sa force d'impact, c'était cette capacité des véritables artistes de créer à partir du désaccord, de la dysharmonie - ou, pour parler autrement, de parvenir à faire œuvre avec ce qui devrait, au contraire, les anéantir » (Lambert 2015, 86).

Si le secret du suicide de Staël à Antibes lui appartient pleinement, s'il est peut-être lié à l'une de ses ruptures sentimentales ou bien à ce vertige que pointe Stéphane Lambert, il reste que les derniers moments de sa vie soulèvent des questions. Car c'est curieusement lorsque le succès arrive, lorsque l'argent afflue, lorsqu'on réclame partout ses toiles, que la dynamique de création se brouille, qu'il ne trouve plus de sens à ce qu'il fait et va mettre fin à ses jours. Comme si le but poursuivi lui avait tragiquement échappé. 


\section{Dépassement, corporéité, gratuité}

Nous aurions pu bien sûr parler de Nicolas de Staël pour aborder les thèmes du désir de reconnaissance et de la mort volontaire comme affirmation personnelle. Nous préférons voir davantage en creux chez lui le signe de trois autres attentes de nos contemporains en termes de dépassement de soi, de corporéité et de gratuité. Trois demandes qui peuvent sembler surprenantes au regard de l'expérience d'un peintre qui nous touche par sa grande liberté face aux cadres établis, voire aux modes.

Même si la vie de Staël s'achève d'une manière tragique, comment nier cependant qu'il laisse une ouvre, une trace forte, et traduit aussi ce désir de réalisation de soi qui habite nombre d'entre nous? Comme s'il fallait se dépasser, aller jusqu'au bout d'une quête qui permet d'échapper au vertige et permet de donner le meilleur de soi-même. Faire preuve d'une exigence si haute qu'elle peut conduire à créer et à se consumer à nouveau. Cette attitude pourtant revêt une ambiguité qu'il convient d'interroger.

Car que peut signifier cette notion de dépassement pour nombre de nos contemporains? Loin de la quête artistique que nous venons de décrire, cette réalisation de soi semble passer désormais par les chemins de ce qu'on nomme épanouissement, développement ou accomplissement personnel. Avec la sécularisation et l'atténuation voire la disparition des croyances religieuses, avec l'insistance sur l'autonomie individuelle, il s'agit de trouver sa propre voie de bonheur. Dans ce projet, la dimension psychologique apparaît très présente, les émotions se révèlent déterminantes et le travail sur soi invite à se libérer des liens qui entravent à travers pesanteurs et histoires personnelles. Après d'autres, relevons que cette quête de performance individuelle n'est pas étrangère à l'idéologie transhumaniste de l'homme augmenté, déjà signalée. De même qu'il nous faut dépasser la précarité du corps, il faut atteindre ici une sorte de solidité psychique qui doit pouvoir aider à affronter les difficultés de la vie. Cette demande de développement personnel ou même spirituel, on la voit fleurir dans les rayons des librairies, qu'elle s'exprime en termes de recherche d'équilibre, de bien-être ou d'épanouissement de soi, par des techniques de méditations ou psychologiques offrant toutes sortes de réponses qui se veulent « positives ». En ce domaine, la production livresque a largement supplanté les ouvrages religieux et grignote souvent aussi le domaine philosophique et la littérature plus classique. 
Pourtant, si elle peut paraître $a$ priori légitime, si elle entend se substituer à des morales ou des attitudes religieuses qui ont souvent contribué à étouffer l'individu, à contraindre ses aspirations profondes, cette quête de la réalisation personnelle aboutit bien souvent à ne pas vraiment prendre en compte notre propre fragilité, à insister sur un développement individuel supposé infini, tout en niant aussi le plus souvent la dimension d'altérité. Car au fond, ai-je besoin des autres pour accéder à ma propre vérité et me déployer pleinement? N'est-ce pas d'abord mon propre moi qui compte? Ai-je besoin de me confronter à ce qui résiste, que ce soient mes limites, ma propre mort ou la présence de tous ceux qui m'entourent?

En ce sens, si certains ont cru bon de rapprocher, voire de concilier développement personnel et christianisme, si par refus compréhensible d'une approche rigoriste il a fallu réaffirmer l'importance d'une certaine recherche du bonheur, il n'empêche que ce souci de concorde bute toujours sur l'affirmation de la faiblesse humaine et de la présence de la Croix. Comment faire fi en effet de la capacité humaine à faire le mal et de la condition mortelle? Peut-on éviter d'affronter son propre "vertige »? À travers une série de portraits où il évoquait de sa plume acérée Pascal, Molière et Racine, François Mauriac rappelait cette nécessaire acceptation de sa propre fragilité issue de la tradition chrétienne : «C'est une étrange injustice que de faire grief au chrétien du sentiment qu'il a de sa propre misère, et de l'accuser d'être faible parce qu'il se connait comme tel. Il n'y a pas de courage à ne pas se connaître; mais il y en a beaucoup à se regarder en face » (Mauriac 1949, 34-35).

Attente de réalisation de soi, donc. Mais aussi quête de corporéité, désir de s'exprimer à travers la chair, le toucher, la matière. Les biographes insistent, il y a chez Nicolas de Staël une débauche d'utilisation de matières, de tubes de couleurs, et cette débauche n'est pas à l'époque sans choquer ses amis peintres durant une période marquée par la guerre et la pénurie de moyens. Staël qui n'en a que faire continue à créer sans cesse en dépensant. Faut-il voir dans cette attitude une manière de s'incarner davantage, de trouver forme? Sans s'enfermer dans la vaine querelle entre abstrait et figuratif, le peintre s'attellera ainsi durant toute une période (1951-1955) à une série de nus, qui visent au plus profond à «faire corps ». Comme l'écrit à son sujet Maryline Desbiolles : «Toute une vie de peintre pour faire apparaître d'un trait mon vis-à-vis, pour le sortir du flou, de l'indifférencié, sa chair non pas dissoute par le coup de crayon mais induite par lui, sa chair 
menacée de disparition, au premier chef, et désirable » (Desbiolles 2014, 87).

Nous voici donc revenus au corps, à la chair vulnérable, à cette incarnation qui ne cesse de nous tarauder. Mais tel Janus le dieu biface, ce corps semble exprimer des attentes spirituelles bien différentes, opposées peut-être.

Parmi les phénomènes contemporains, ceux de la course et de la marche ne traduisent-ils pas cette forme de dépassement de soi qui passe aussi par le corps, mais un corps qui somme toute va bien et obéit, parfois jusqu'à l'extrême? Ainsi ceux qui courent durant leur temps de loisir, qui n'hésitent pas à traverser l'Atlantique pour suivre le marathon de New York, pourraient fort bien se livrer à la même activité dans la vieille Europe. Est-ce pour eux une simple occasion de se détendre et de rechercher la performance personnelle à tout prix, ou bien de se déconnecter, voire de vivre un temps de solitude face à soi-même et à ses propres limites ? De s'éprouver, en quelque sorte.

Et puis, il y a les marcheurs, tous ceux qui choisissent volontairement de consacrer du temps à la marche dans un monde où les moyens de transport sont de plus en plus rapides. Giacometti n'a-t-il pas été un peu prophète, à sa manière, en sculptant L'homme qui marche? Dans son roman L'bomme qui fuyait le Nobel, Patrick Tudoret met ainsi en scène un écrivain qui a choisi de quitter le monde en se lançant sur le chemin de Compostelle, une voie empruntée par beaucoup, et pas seulement pour des raisons religieuses ou spirituelles explicites (Tudoret 2015). « À la différence de la promenade ou de la balade, la marche est engageante », explique de son côté Gaële de la Brosse dans une enquête réalisée auprès de ceux qui la pratiquent.

Par ses détours et ses impasses, elle déstabilise, rend vulnérable. Cependant, en renouvelant la grille de lecture du paysage comme de la vie, elle est résolument optimiste : même si elle n'apporte pas de réponse à toutes les questions, elle propose des horizons, montre des pistes. À ceux qui ont perdu le fil de l'existence, elle esquisse une nouvelle trame. Que l'on se dirige vers un sanctuaire, une ville mythique ou un sommet, la marche redonne saveur à l'existence. (de la Brosse 2019, 20)

Au fond, pratiquer une fragilité volontaire pour mieux se fortifier ensuite. 
Mais vient aussi le temps où le corps ne répond plus, où la maladie, la vieillesse ou la mort prennent le dessus. Il n'est plus question dès lors de courir et de marcher, de viser la performance, mais de se recentrer sur les besoins essentiels. Sur le lit du malade ou du mourant, voici le corps à nouveau omniprésent, non plus performant et tendu, mais exposé et offert, comme lâchant prise. Voici le souffle qui ne fonctionne plus comme une machine efficace mais se fait toute ténuité et fragilité, guetté en permanence par l'entourage. Voici le corps appelant plus qu'avant le soin et l'attention des autres, à travers les demandes les plus élémentaires. Or, c'est à travers cette vulnérabilité que peuvent s'exprimer les attentes spirituelles ultimes. Attentes d'un peu d'eau, de fraîcheur ou de draps propres, mais qui disent plus foncièrement une demande de paix, d'amour et de dignité, d'assurance aussi face à l'inconnu de ce qui va arriver. Soif qui n'est pas seulement un besoin physiologique, mais ouverture à un mode de relations et de dépendances inédit. Il n'est plus temps alors de se dépasser soi-même mais de consentir au soin, à une forme nouvelle d'abandon, à une sorte de réconciliation aussi peut-être. Si cette autre image du corps frêle peut faire peur à nos contemporains, ceux-ci ne peuvent en faire aussi facilement l'économie au cours de leur existence. On ne peut mettre indéfiniment l'incarnation, «le corps de chair », sous le boisseau (Fiat 2015).

À côté de cette attente de réalisation de soi, d'incarnation, il faut signaler aussi l'attente de gratuité. Elle est éminemment présente dans le soin apporté au malade et aux mourants que nous venons rapidement d'évoquer. À sa manière, Nicolas de Staël nous interroge sur cette dimension. Il est en effet surprenant de constater que lorsqu'il connait le succès, lorsque la réussite matérielle arrive enfin, sa démarche de création semble ne plus fonctionner. Deux logiques semblent ne plus correspondre dès lors, comme si l'argent ou l'utilité économique avaient cassé un élan intérieur plus fort. N'avons-nous pas nous aussi complètement intégré cette démarche d'utilité, de rentabilité ou d'efficacité qui fait que nous peinons à trouver en nous des sources de générosité ou de simple respiration? Au cœur d'emplois du temps trop chargés, rendus plus performants grâce à des moyens techniques très puissants, soucieux à la fois de travail et de loisir tout aussi « rentabilisés », quelle place laissons-nous encore à la contemplation et à la beauté? Acceptons-nous de nous arrêter un moment pour lire un texte, admirer un tableau ou une œuvre musicale, voire simplement parler à notre semblable? 
On comprend du coup l'insistance faite par Jean-Marc Barreau, dans le cadre de l'accompagnement des personnes en soins palliatifs, sur la conversion mentale, voire spirituelle, qui doit s'opérer chez celles-ci. Il s'agit alors de passer d'une vision de l'existence fondée sur le facere, le faire, l'utilité, à une perception davantage appuyée sur l'agere, la relation au semblable, l'amour, l'attention (Barreau 2017). Ne s'agit-il pas ici de répondre à une véritable attente de gratuité en la faisant émerger? De passer d'une fragilité initiale à une vulnérabilité consentie et partagée?

Achevons notre parcours avec les artistes qui témoignent de notre fragilité avec le musicien et théoricien Glenn Gould.

\section{Les Variations Glenn Gould}

Peindre des footballeurs n'a pas empêché Nicolas de Staël de représenter aussi des musiciens dans ses toiles les plus célèbres comme Le concert et ses différents Pianos. Est-ce à dire que la musique est aussi le lieu où peuvent s'exprimer nos propres fragilités, par son caractère insaisissable et ineffable (Jankélévitch 1983) ? Regardant les pianos noirs de Staël, je ne peux m’empêcher de penser aussi à l'instrument de Glenn Gould (1932-1982). Si l'on continue à écouter autant aujourd'hui la musique de Jean-Sébastien Bach, si une pièce de virtuosité aussi complexe que Les variations Goldberg est devenue une œuvre populaire, c'est en grande partie grâce à l'interprétation de Glenn Gould qu'on le doit. Curieusement, son jeu tout en staccato, si peu lié, plus proche de l'esthétique du jazz que celle du renouveau baroque, a su trouver les faveurs d'un public très large. Mais la grande clarté du jeu, qui peut paraître à priori, sec, froid permet au contrepoint de se développer et de mettre en valeur toutes les voix écrites par le compositeur.

Si même longtemps après sa mort le personnage continue de fasciner, c'est sans doute, comme pour Staël, dans le lien étroit qu'il établit entre l'art et la vie, mais aussi parce que cette quête du beau se joue dans un corps et une psychologie toute en fragilité. À ce sujet, on a pu parler d'hypocondrie devant sa phobie des maladies ou du froid, voire même de syndrome d'Asperger qui l'a conduit à se couper du monde et à renoncer définitivement aux concerts publics. Comment expliquer en effet le contraste entre le génie de Gould, son succès mondial, sa capacité à restituer la force d'une œuvre comme celle de Bach et la réalité d'une existence qui ne se sent en rien assurée, toujours en inquiétude, tentée par le retrait? Dans 
le très bel essai qu'il lui a consacré, Georges Leroux, n’hésite pas à parler à son sujet d'un «don de soi " pour la recherche du contrepoint absolu, en signalant à sa manière ce paradoxe : «Un des traits les plus vifs de l'art et de la vie de Glenn Gould est justement cet engagement absolu dans la vie de l'art et cette reconnaissance des limites de la forme de vie de l'art » (Leroux 2007, 47).

Si Gould a pu se référer au Dernier puritain du philosophe Santayana, sa démarche de renoncement à soi-même peut sans doute être rapprochée davantage des modèles de sainteté plus classiques. Michel Schneider n'hésite pas à écrire de son côté : "Dans son studio, Gould renouait avec cet idéal monastique du status religiosus, vie de renoncement et chemin de perfection dont les pas sont pauvreté, chasteté et obéissance. Son approche de la musique est du même ordre que celle de Dieu pour les mystiques » (Schneider 1988, 23).

Il reste que cette vie de retrait et de solitude ne conduit pas à garder l'œuvre pour soi, mais bien à l'offrir au plus vaste. «L'artiste [écrit Georges Leroux], partage avec le saint ce renoncement à l'œuvre, puisque la quête de l'œuvre est d'emblée portée par le don » (Leroux 2007, 51). On comprend mieux pourquoi, après que le pianiste ait mis fin à sa carrière de concertiste par refus de la «starisation », celui-ci va se consacrer à des tâches de communication beaucoup plus larges. Le disque, les enregistrements et les émissions vont lui permettre de développer davantage cette capacité de don, et ce jusqu'à aujourd'hui, par-delà sa propre disparition.

"Seigneur, donnez-nous la paix que la terre ne peut nous donner », priait-on lors des offices religieux auxquels le jeune Gould assistait dans sa jeunesse. Même s'il ne pratiquait plus depuis longtemps, il continuait semble-t-il de se répéter cette incantation (Schneider 1988, 159). Mais en quoi est-il lui aussi révélateur d'autres attentes spirituelles contemporaines?

\section{$7 \quad$ Ritualité, nature et images de Dieu}

Tremper ses mains à l'eau bouillante avant de jouer, garder toujours la même chaise confectionnée par son père devant le piano, choix précis des vêtements, on sait tout le prix qu'accordait Glenn Gould à de tels rituels. Manies de diva, exercices de concentration, voire obsessions, pourraient dire certains? C'est oublier que le travail de l'artiste nécessite une forme 
d'ascèse et de mise en condition. Chaque jour, il faut remettre son ouvrage sur le métier, ce que Matisse appelait « aller au feu ». Rite infini du travail de l'œuvre, toujours repris, toujours recommencé, même s'il ne se passe rien parfois. À sa manière, Gould nous interroge sur la demande de ritualité présente chez nombre de nos contemporains, au-delà de la seule expérience artistique.

Comment appréhender cette demande de ritualité dans une culture où la plupart des cadres confessionnels ont disparu? Si elle peut apparaître de manière explicite dans certaines formes du retour du religieux, par exemple, qu'on pense aux jeunes qui se convertissent à un islam très rigoriste ou à ses séminaristes catholiques qui accordent une place très importante aux formes liturgiques, elle se dévoile aussi à travers des expressions plus sauvages, spontanées. Pensons ainsi à ces fleurs que l'on pose sur le lieu d'un accident mortel, à ces temps de convivialité ou de partage qu'on s'accorde après un deuil ou un événement plus heureux. Alors qu'ils n'étaient plus guère en vigueur, les rites de fiançailles ou «d'enterrement de la vie de garçon ou de jeune fille » ont curieusement refait surface dans des sociétés où le mariage n'est plus un modèle familial dominant. Et, de manière paradoxale, on voit les communautés homosexuelles obtenir le droit de se marier alors qu'il y a peu, certains membres d'entre elles rejetaient l'institution du mariage, jugée trop «bourgeoise » ou « dépassée ».

Si la demande de ritualité se révèle toujours si prégnante, c'est sans doute pour mieux répondre à la fragilité des individus en leur offrant un cadre qui rassure. N'est-ce pas renouer là avec une réalité anthropologique forte, lutter contre la mort et l'implacable écoulement du temps, tenter de fournir un peu de sens au cœur d'une réalité qui nous échappe? Il s'agit alors de structurer le quotidien, de donner de l'unité dans une vie ordinaire déjà fortement éclatée, en proie aux multiples distractions, divertissements, mais aussi pesanteurs et sollicitations comme celles de moyens de communication très présents. Si la messe du dimanche ne concerne plus qu'un public restreint, bien des habitudes de retrouvailles familiales demeurent. Au cœur de modes de vie souvent frénétiques, le rite permet une pause salutaire.

À côté de cet ordinaire des jours, le rite offre également de marquer les moments extraordinaires, devenus sans doute plus rares dans nos temps de confort et de sécurité matérielle, où guerres, épidémies ou saisons ne rythment plus le cours de la vie sociale. Il demeure aussi le moyen privilégié 
de saluer davantage les grandes étapes de l'existence - naissance, anniversaires, amour, mort... - comme autant d'occasions d'arrêt, de pause qui sont encore là pour célébrer mais également susciter des brèches, des lieux de réflexion, de partage ou d'intériorisation. Les accompagnants des personnes en fin de vie savent ce qu'il en est de l'importance des rites, à condition bien sûr que ceux-ci permettent un vrai travail d'humanisation et ne dégénèrent pas en ritualisme.

Autre attente d'ordre spirituel dont témoigne Gould et que l'on voit se déployer aujourd'hui très largement, le lien à la nature. Sans considérer le musicien comme un chantre de l'écologie avant l'heure, on trouve chez lui ce trait, remarque Georges Leroux, qui en souligne d'ailleurs la dimension canadienne : "Cet amour immédiat et constant de la nature, telle qu'elle s'offre dans cette campagne tranquille, c'est le rêve de tous les Canadiens industrieux, comme si elle leur était offerte en récompense de leur effort, comme si elle était la vérité de l'existence au Canada »(Leroux 2007, 304). Cet attachement à la nature de Gould — qui se traduit dans la relation aux grands espaces, au Nord, aux lacs et aux arbres - puise, semble-til, au transcendantalisme de Whitman ou de Thoreau. Notons qu'il est aujourd'hui beaucoup plus universel que canadien et habite bien des aspirations actuelles.

Sans pour autant épouser les mythes du New Age - qui voient dans Gaïa la déesse-mère source d'énergie, sans parler « d'harmonie cosmique » ni de fusion dans une sorte de grand Tout - , il est indéniable que la nature continue de porter par elle-même tout un imaginaire de l'intériorité. Qu'elle soit montagne enneigée ou paisible campagne, désert aride ou océan infini, celle-ci apparaît toujours comme un lieu de silence et de paix, occasion d'un ressourcement bienvenu. Elle vient combler autant les attentes du marcheur que celle de l'individu en quête de solitude. À la suite des Psaumes ou de François d'Assise, le croyant y verra la trace d'une Création divine, et l'agnostique un surprenant agencement de formes, de matières et de couleurs. Que l'on croie ou non d'ailleurs, l'image du ciel étoilé renvoie peu ou prou à la notion d'infini.

Reste pourtant que cette belle image semble singulièrement s'altérer en ce début de millénaire. C’est que la nature, les ressources de la Terre, l'infini du monde apparaissent comme autant d'entités menacées. De rassurant qu'il pouvait être, l'espace naturel fait figure lui aussi de fragilité, de 
ce lieu où vacille tout un pan de notre identité humaine et spirituelle. Réchauffement du climat, pollution, disparition de forêts, épuisement de nombre de ressources, les tristes empreintes de l'homme manifestent à la fois sa tentation de toute-puissance et son extrême vulnérabilité. Que connaitront demain de la planète nos petits-enfants, nous qui voyons disparaître les glaciers de notre enfance? Ne faut-il pas craindre la catastrophe ultime? L'humanité pourra-t-elle survivre à la destruction de son milieu? L'attente spirituelle se fait ici angoisse et peur.

Il faut venir enfin à une dernière attente qui viendra clore cet article et que l'on ne peut qu'aborder avec prudence. À propos de Glenn Gould, Michel Schneider n'hésite pas à écrire que : « Derrière ses consoles de métal et son piano noir, il faisait ce que peu d'artistes font encore : au-delà de son art, il dévisageait Dieu » (Schneider 1988, 223). À travers leurs recherches spirituelles, nos contemporains tentent-ils à leur manière de "dévisager Dieu »? Et de quel Dieu s'agit-il ? Peut-on rendre compte un tant soit peu de ce sentiment religieux souvent intime, à un moment où se croisent religions, traditions, philosophie dans un contexte de dialogue interculturel inédit? Existe-t-il une véritable attente en termes d'altérité divine?

Même au sein de religions instituées, il devient difficile pour nombre de nos contemporains d'enfermer Dieu dans les catégories du dogme. La foi passe d'abord au crible de la vérification personnelle, d'une expérience qui n'entend pas se faire imposer sa propre croyance. D'une étude dirigée voici quelques années par le sociologue Jean-François Barbier-Bouvet auprès des personnes qui fréquentent des centres spirituels se réclamant de différentes traditions, il ressort fortement que la figure du Dieu personnel, chère aux monothéismes, laisse souvent place à une figure plus indéterminée et impersonnelle (Barbier-Bouvet 2015). Parler du divin en soi désigne moins sans doute alors un Être bien défini qu'une présence, voire une forme d'énergie à laquelle il est possible de se ressourcer. Si l'on excepte les tenants les plus convaincus des grandes traditions, il semble bien que les individus contemporains soient devenus plus méfiants vis-àvis d'un Dieu qui se présentait par trop extérieur et transcendant. Pour être crédible, il lui faut rejoindre le cœur et les émotions. On comprend mieux alors l'engouement actuel pour la pratique de la méditation qui invite à se rendre plus disponible à soi-même, à ce qui est présent, plutôt qu'à s'adresser à une entité divine, à une altérité. Mais alors, quelle place des autres, et de l'Autre, dans tout cela? 
Même largement partagée, cette figure du divin sera-t-elle en mesure de répondre vraiment aux attentes spirituelles de l'homme fragile d'aujourd'hui et de son cœur sans repos? La question mérite au moins d'être entendue.

\section{Conclusion}

À partir de l'expérience de la fragilité perçue à travers trois artistes contemporains, Sylvie Germain, Nicolas de Staël et Glenn Gould, nous avons tenté de dégager quelques grandes attentes spirituelles présentes dans les mentalités et la culture d'aujourd'hui. Celles-ci interpellent principalement tous ceux qui, à des titres divers, sont engagés dans le domaine du soin et de l'éthique, obligeant aussi à mieux formuler notre langage religieux, voire théologique. Les demandes de reconnaissance, d'écoute et d'éternité invitent à passer d'une simple considération du Moi à une meilleure évaluation de ses aspirations profondes. Les attentes sur les plans du dépassement de soi, de la corporéité et de la gratuité conduisent à passer d'une culture de la seule performance à un vrai souci de la dignité de la personne. Enfin, les requêtes en termes de ritualité, de sens de la nature et de nomination de Dieu sont autant de stimulations pour des pratiques d'accompagnement pastoral et des présentations renouvelées de l'espérance chrétienne.

Loin de toute attitude de résignation, notre conscience de la fragilité invite tout au contraire à l'imagination. Le défi n'est-il pas de répondre à l'inquiétude qui nait de la fragilité par des œuvres de soin, de culture et d'ouverture spirituelle?

\section{Références}

ARÈnES, J. (1999), Accueillir la faiblesse, Paris, Desclée de Brouwer.

Augustin (Saint) (1964), Confessions / trad. par J. Trabucco, Paris, Garnier-Flammarion.

BARBIER-BOUVET, J.-F. (2015), Les nouveaux aventuriers de la spiritualité. Enquête sur une soif d'aujourd'hui, Paris, Mediaspaul.

Barreau, J.-M. (2017), Soins palliatifs, Accompagner pour vivre, Montréal, Médiaspaul.

Bellet, M. (1989), L'écoute, Paris, Epi-Desclée de Brouwer. 
Brosse, G. de la (2019), Le petit livre de la marche, Paris, Salvator.

Benasayag, M. (2004), La fragilité, Paris, La Découverte.

CARrière, J.-C. (2006), Fragilité, Paris, Odile Jacob.

Chrétien, J.-L. (2017), Fragilité, Paris, Éditions de Minuit.

Desbiolles, M. (2014), «Écrire comme un peintre », dans J.-L. Andral, dir., Staël, la figure à nu, 1951-1955, Paris, Hazan (Catalogues d'exposition).

FIASSE, G. (2015), Amour et fragilité. Regards philosophiques au cour de l'humain, Québec, Presses de l'Université Laval.

FIAT, E. (2015), Corps et âme. Ou qu'un peu d'incarnation ça peut pas faire de mal..., Nantes, Éditions Nouvelles Cécile Defaut.

Germain, S. (1985), Le Livre des nuits, Paris, Gallimard. (1989), Jours de colère, Paris, Gallimard. (2000), Cracovie à vol d'oiseaux, Monaco, Éditions du Rocher. (2008), L'inaperçu, Paris, Albin Michel. (2009), Hors champ, Paris, Albin Michel.

GRAND'MAISON, J. (2013), Une spiritualité laïque au quotidien, Montréal, Novalis.

HonneTH, A. (2000) [allemand 1992], La lutte pour la reconnaissance, Paris, Cerf.

Jollien, A. (2011), Éloge de la faiblesse, Paris, Marabout.

KANDINSKY, W. (1989), Du spirituel dans l'art, et dans la peinture en particulier / trad. par N. Debrand et B. Du Crest, Paris, Gallimard (Folio Essais, 72).

Kourilsky, J. (2019), De la science et de la démocratie, Paris, Odile Jacob.

LAMBert, S. (2015), Nicolas de Staël. Le vertige et la foi, Paris, Arléa.

LAUPIES, F. (2019), Sagesse du désir, Paris, Salvator.

LebOUCHer, M. (2017), Le souffle et le roseau. Variations sur la fragilité, Paris, Salvator. 
(2019), «L'irruption de la vulnérabilité vue aux plans sociétaux et médiatiques", dans L. VILLEMIN et C. FiNO, Vulnérabilités. Relecture critique à la croisée des disciplines, Paris, Cerf.

Leroux, G. (2007), Partita pour Glenn Gould. Musique et forme de vie, Montréal, Les Presses de l'Université de Montréal.

MAubert, F. (2016), L'homme qui marche, Paris, Fayard.

MAURIAC, F. (1949), Mes grands hommes, Monaco, Éditions du Rocher.

Pelluchon, C. (2011), Éléments pour une éthique de la vulnérabilité. Les hommes, les animaux, la nature, Paris, Cerf.

Ringlet, G. (2007), Éloge de la fragilité, Paris, Albin Michel.

RoustanG, F. (2019), Le troisième homme. Entre rupture personnelle et crise catholique, Paris, Odile Jacob.

SALENSON, C. (2016), Bouleversante fragilité. L'Arche à l'épreuve du handicap, Paris, Nouvelle Cité.

SCHNEIDER, M. (1988), Glenn Gould piano solo, Paris, Gallimard.

TAYLOR, C. (1998) [anglais 1989], Les sources du Moi. La formation de l'identité moderne / trad. par C. MELANÇON, Paris, Seuil.

Tudoret, P. (2015), L'homme qui fuyait le Nobel, Paris, Grasset.

\section{Résumé}

Si le concept de fragilité prend tout naturellement sa place dans une réflexion d'ensemble sur le soin, il reste qu'il trouve des illustrations dans d'autres secteurs de la culture contemporaine. Écrivains, peintres ou musiciens traduisent à leur manière des attentes spirituelles d'aujourd'hui. Après avoir précisé la notion d'attente spirituelle en lien avec l'inquiétude actuelle, il s'agit ici d'interroger l'œuvre de trois artistes pour mieux les relier à des aspirations plus larges. Si la romancière Sylvie Germain aide à mieux percevoir de telles attentes en termes de reconnaissance, d'écoute et d'éternité de la part des individus d'aujourd'hui, les toiles et la vie de Nicolas de Staël aident de leur côté à discerner celles-ci en termes de dépassement de soi, de corporéité et de gratuité. Le destin du pianiste Glenn 
Gould aide enfin à mieux percevoir ces demandes en termes de ritualité, de relation à la nature et de nomination du divin qui s'expriment désormais.

\section{Abstract}

If the concept of frailty comes up naturally in a general overview of caregiving, illustrations can nonetheless be found in other areas of contemporary culture. Writers, painters and musicians have their own ways of translating today's expectations of spirituality. After having narrowed down the notion of spiritual expectations in relation to today's anxieties, an analysis of the works of three artists will be discussed in order to link them to larger aspirations. If novelist Sylvie Germain helps bring out a modern-day individual's expectations in terms of recognition, listening and eternity, the paintings of Nicolas de Staell, for their part, help clarify these notions in terms of self-transcendence, corporeality, and gratuitousness. Finally, the destiny of pianist Glenn Gould helps show these demands in terms of today's expressions of rituality, relations with nature and the nomination of the divine. 